\title{
Spin density wave in oxypnictide superconductors in a three-band model
}

\author{
Mingsheng Long, Liangbin $\mathrm{Hu}, \mathrm{W}$. LiMing* \\ Dept. of Physics, and Institute for Condensed Matter Physics, \\ School of Physics and Telecommunication Engineering, \\ South China Normal University, Guangzhou 510006, China
}

(Dated: November 17, 2018)

\begin{abstract}
The spin density wave and its temperature dependence in oxypnictide are studied in a threeband model. The spin susceptibilities with various interactions are calculated in the random phase approximation(PPA). It is found that the spin susceptibility peaks around the M point show a spin density wave(SDW) with momentum $(0, \pi)$ and a clear stripe-like spin configuration. The intraband Coulomb repulsion enhances remarkably the SDW but the Hund's coupling weakens it. It is shown that a new resonance appears at higher temperatures at the $\Gamma$ point indicating the formation of a paramagnetic phase. There is a clear transition from the SDW phase to the paramagnetic phase.

PACS numbers: 74.20.-z, 74.25.Jb, 74.72.-h
\end{abstract}

\section{INTRODUCTION}

The high temperature superconductivity in the newly discovered oxypnictides, $\mathrm{LnFeAsO}(\mathrm{Ln}=\mathrm{La}, \mathrm{Pr}, \mathrm{Ce}, \mathrm{Sm}$ ), has attracted great attention aiming to identify the mechanism of superconductivity in these materials 1]. Recently the transition temperature $T_{c}$ is dramatically raised from $26 K$ to $43 K[2]$. In addition to the high $T_{c}$, these materials display many other interesting properties. The most interesting phenomena is the presence of competition between the magnetically ordered ground states [3] of spin density wave(SDW) and superconductivity, but a controversy model explains the superconductivity by means of antiferromagnetic spin fluctuation in $\mathrm{LaFeAsO}$ 4. Pure oxypnictide is not superconducting but shows an anomaly at about $150 \mathrm{~K}$ in both resistivity and dc magnetic susceptibility [1, 5]. Both experimental and theoretical evidences show that the anomaly is caused by the SDW instability [3, 6, 7].

It is shown by the first principle calculations that the band structure of LnFeAsO near the Fermi surface (FS) is formed by a hole-like pocket centered around the $\Gamma$ point and an electron-like pocket around the $\mathrm{M}$ point in the extended Brillouin zone(BZ)(one Fe atom per unit cell) [8 10]. A strong FS nesting effect exists between the hole and electron pockets with commensurate wave vectors, $(\pi, 0)$ and its symmetric ones. This leads to a strong SDW instability, and is believed to cause the the anomaly at $150 K$.

Raghu et al calculated the SDW in the iron oxypnictides within a minimal two-band model in the phase random approximation(RPA). They found that the SDW is enhanced significantly by the intra-band Coulomb repulsion. The influence of the inter-band Coulomb interaction and the Hund's coupling, however, have not yet been fully studied in literatures. Different researchers se-

*Electronic address: Corresponding author: wliming@scnu.edu.cn

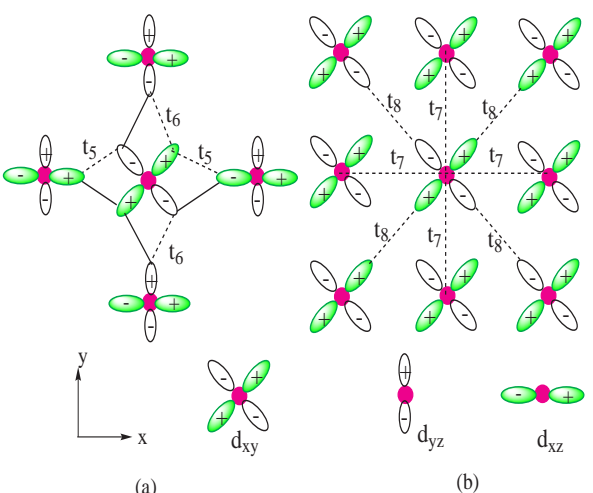

FIG. 1: (a) A schematic diagram showing the hopping parameters between $d_{x z}\left(d_{y z}\right)$ and $d_{x y}$. (b) The hopping parameters between the nearest and next nearest $d_{x y}$ orbitals.

lect different groups of interaction parameters, but the relation between them has not been revealed. In addition, the temperature dependence of the SDW has hardly been theoretically considered. As pointed out by Lee and Wen 11], a three-band model reproduces more accurately the band structure near the Fermi surface of oxypnictides. In this work we study the SDW in a three-band model in the RPA and the temperature dependence of the spin susceptibility. The magnetic instability is studied for a wide range of interaction parameters. We found a new resonance of the spin susceptibility around the $\Gamma$ point at higher temperatures, indicating the formation of a paramagnetic phase. There appears a transition from the SDW phase to the paramagnetic phase when temperature increases.

\section{MODEL HAMILTONIAN}

The FeAs layer in LaFeAsO forms a square lattice, where $\mathrm{Fe}$ ions locate on the lattice sites and an As ion sits at the center of each square. Various band structure 
calculations showed that the main contribution to the density of states near the FS comes from $d_{x z}, d_{y z}$ and $d_{x y}$ of the five $3 d$ orbitals of Fe atoms [11]. The left two orbitals are far apart from the FS.

In the three band model, the hopping terms between $d_{x z}, d_{y z}$ and $d_{x y}$ as illustrated in Fig. 1 are included in the Hamiltonian. They are written as [11, 12]

$$
H_{0}=\sum_{\mathbf{k} \sigma} \Psi_{k \sigma}^{\dagger} M_{\mathbf{k}} \Psi_{k \sigma}
$$

where the three-component field $\Psi_{k \sigma}$ is defined as $\Psi_{k \sigma}=$ $\left(d_{x z \sigma}(\mathbf{k}), d_{y z \sigma}(\mathbf{k}), d_{x y \sigma}(\mathbf{k})\right)^{T}$ and the Matrix $M_{\mathbf{k}}$ is given by

$$
M_{\mathbf{k}}=\left(\begin{array}{lll}
\varepsilon_{11}(k) & \varepsilon_{12}(k) & \varepsilon_{13}(k) \\
\varepsilon_{21}(k) & \varepsilon_{22}(k) & \varepsilon_{23}(k) \\
\varepsilon_{31}(k) & \varepsilon_{32}(k) & \varepsilon_{33}(k)
\end{array}\right),
$$

with elements

$$
\begin{aligned}
& \varepsilon_{11}=-2 t_{1} \cos k_{x}-2 t_{2} \cos k_{y}-4 t_{3} \cos k_{x} \cos k_{y} \\
& \varepsilon_{22}=-2 t_{2} \cos k_{x}-2 t_{1} \cos k_{y}-4 t_{3} \cos k_{x} \cos k_{y} \\
& \varepsilon_{33}=-2 t_{7} \cos k_{x}-2 t_{7} \cos k_{y}-4 t_{8} \cos k_{x} \cos k_{y} \\
& \varepsilon_{12}=\varepsilon_{21}=-4 t_{4} \sin k_{x} \sin k_{y} \\
& \varepsilon_{13}=-\varepsilon_{31}=-2 i t_{5} \sin k_{x} \\
& \varepsilon_{23}=-\varepsilon_{32}=-2 i t_{6} \sin k_{y} .
\end{aligned}
$$

The hopping parameters are set to $t_{1}=-1.0(\approx$ $0.4 \mathrm{eV}), t_{2}=0.7, t_{3}=-0.80, t_{4}=0.6, t_{5}=t_{6}=$ $-0.35, t_{7}=-0.3, t_{8}=0.2, \mu=1.15$, in units of $\left|t_{1}\right|[12]$.

The energy dispersion is plotted in Fig. 2(a) in the extended BZ and 2(b) along the $\Gamma \rightarrow X \rightarrow M \rightarrow \Gamma$ in the folded BZ(two Fe atoms per unit cell). Fig. 2(d) plots the density of states of the band structure. It is seen that there are two dominating Van Hove singularities near the Fermi level, close to the hole and electron pockets. The density of states is significantly different from the twoband model, of which the Van Hove singularities are more distant from the Fermi level. A stronger nesting effect is expected in this three-band model. The third band is mixed with the two conventional bands and thus should have great contribution to the spin susceptibility.

\section{THE SPIN SUSCEPTIBILITY}

The static spin susceptibility in the non-interacting case is given by $\chi^{(0)}(\mathbf{q})=\sum_{l l^{\prime}} \chi_{l l^{\prime}}^{0}(\mathbf{q})$ with

$$
\chi_{l l^{\prime}}^{0}(\mathbf{q})=\frac{1}{2 N} \sum_{k} \frac{f\left(\varepsilon_{\mathbf{k} l}\right)-f\left(\varepsilon_{\mathbf{k}+\mathbf{q} \mathbf{l}^{\prime}}\right)}{\varepsilon_{\mathbf{k}+\mathbf{q}^{\prime}}-\varepsilon_{\mathbf{k} l}}\left|<k+q, l^{\prime}\right| k, l>\left.\right|^{2}(3)
$$

where $l, l^{\prime}=1,2,3$ are band indexes, $f(\varepsilon)=1 /\left(e^{\beta(\varepsilon-\mu)}+\right.$ 1 ) is the Fermi distribution function, $\beta=1 / k T$, and $\varepsilon_{\mathbf{k} l}$ and $\mid \mathbf{k}, l>$ are the eigenvalues and eigenvectors respectively of the Hamiltonian matrix (2).

We fix $k T=0.02(\sim 93 K)$ for a finite lattice with $64 \times$ $64 \mathrm{k}$ meshes in the extended BZ to calculate the static
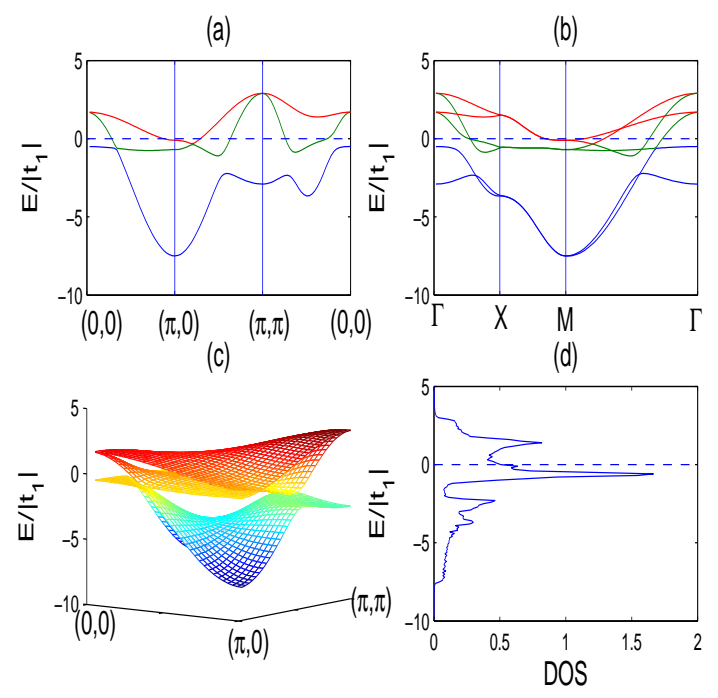

FIG. 2: (a) Energy dispersion in the unfolded BZ of the threeorbital model with $t_{1}=-1.0, t_{2}=0.7, t_{3}=-0.80, t_{4}=$ $0.6, t_{5}=t_{6}=-0.35, t_{7}=-0.3, t_{8}=0.2, \mu=1.15$ along the path $(0,0) \rightarrow(\pi, 0) \rightarrow(\pi, \pi) \rightarrow(0,0)$. (b) Energy dispersion in the folded BZ along $\Gamma=\mathbf{q}=(\mathbf{0}, \mathbf{0}), X=\mathbf{q}=\left(\frac{\pi}{2}, \frac{\pi}{2}\right), M=$ $\mathbf{q}=(\pi, \mathbf{0})$. (c) Energy dispersion on the $\left(k_{x}, k_{y}\right)$ plane. (d) density of states of the three-band model, where the dashed line represents the Fermi level.

spin susceptibility, which is shown in Fig.3(upper). The static spin susceptibility shows great peaks at $(0, \pm \pi)$ and $( \pm \pi, 0)$. This indicates that a collinearly-striped antiferromagnetic (AFM) order phase, a spin density wave (SDW), exhibits in oxypnictide. This feature is in agreement with the neutron scattering measurements [5, 7, 13]. The SDW comes from the strong nesting between the hole pocket at the $\Gamma$ point and the electron pocket at the $\mathrm{M}$ point in the extended BZ. It is observed experimentally that this SDW peak is significantly suppressed by $\mathrm{F}$ doping [3]. This is reasonable because a down-shift of the Fermi level tends to reduce the size of electron pocket and enlarge the hole pocket thus to suppress the nesting between them.

Now we consider the interactions: the intra-band Coulomb repulsion $U$, the inter-band coulomb interaction $U^{\prime}$, the Hund's coupling $J$. The interaction Hamiltonian is written as

$$
\begin{aligned}
H_{i n t} & =U \sum_{i l} n_{i l \uparrow} n_{i l \downarrow}+U^{\prime} \sum_{i, l \neq l^{\prime}} n_{i l} n_{i l^{\prime}} \\
& +J \sum_{i, l \neq l^{\prime}} \mathbf{S}_{i l} \cdot \mathbf{S}_{i l^{\prime}}
\end{aligned}
$$

In the RPA[14], the spin susceptibility, $\chi^{s}(\mathbf{q})$, and the charge-orbital susceptibility, $\chi^{c}(\mathbf{q})$, with interactions are given by

$$
\begin{aligned}
& \chi^{s}(\mathbf{q})=\left[\hat{I}-U^{s} \chi^{0}(\mathbf{q})\right]^{-1} \chi^{0}(\mathbf{q}), \\
& \chi^{c}(\mathbf{q})=\left[\hat{I}+U^{c} \chi^{0}(\mathbf{q})\right]^{-1} \chi^{0}(\mathbf{q}),
\end{aligned}
$$



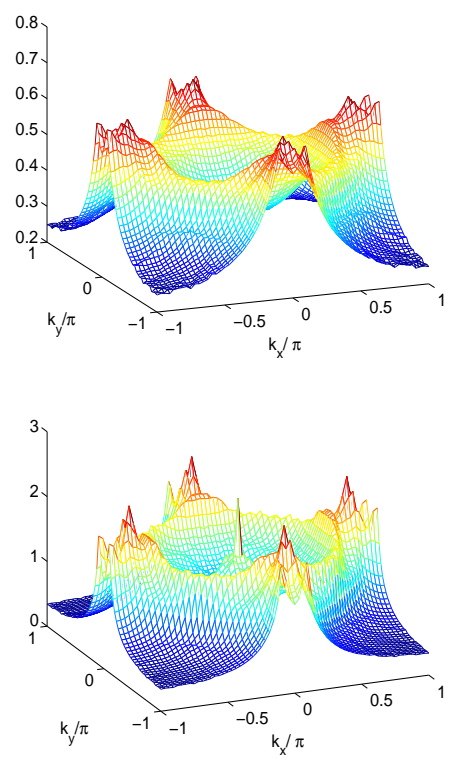

FIG. 3: The static spin susceptibility $\chi^{(0)}(\mathbf{q})$ versus $\mathbf{q}($ upper). The static spin susceptibility $\chi(\mathbf{q})$ in the RPA(lower) with $U=3.0, J=0 ., k T=0.02$.

where $\chi^{0}(\mathbf{q})$ is a $3 \times 3$ matrix with elements defined in (3) and $U^{s(c)}$ are the interaction matrices

$$
U^{s}=\left[\begin{array}{ccc}
U & -J & -J \\
-J & U & -J \\
-J & -J & U
\end{array}\right], U^{c}=\left[\begin{array}{ccc}
U & 2 U^{\prime} & 2 U^{\prime} \\
2 U^{\prime} & U & 2 U^{\prime} \\
2 U^{\prime} & 2 U^{\prime} & U
\end{array}\right]
$$

It is seen from (15) and (6) there will appears a magnetic instability when the following relations are satisfied:

$$
\operatorname{det}\left[\hat{I} \mp U^{s(c)} \chi^{0}(\mathbf{q})\right]=0
$$

To show the magnetic instability at the M point the determinants (7) at the SDW momentum, $(0, \pi)$, are calculated for different interaction parameters and are plotted in Fig.4. The parameters on the contour lines labeled by "-0-" in these two diagrams give zero determinants thus lead to magnetic instabilities. For a fixed $U$ the spin susceptibility decreases with increasing Hund's coupling $J$ thus reduces the SDW peak at the M point. This result is quite different from that made by Raghu et al[15], who found stronger spin fluctuations with increasing $J$. The lower diagram in Fig.3 shows the spin susceptibility close to the magnetic instability with $U=3.0, J=0.0$, where the SDW peak is much enhanced due to the intraband Coulomb repulsion relative to that of the bare spin susceptibility. When $J$ increases, however, the SDW peak drops significantly until the SDW phase fully disappears. It is interesting to notice that, when $U<5$, a ferromagnetic coupling $(J<0)$ is beneficial to the formation of the SDW on the M point. On the other hand, the charge-orbital instability appears in the region $-4.2<U^{\prime}<0$. That is, when a Coulomb attraction
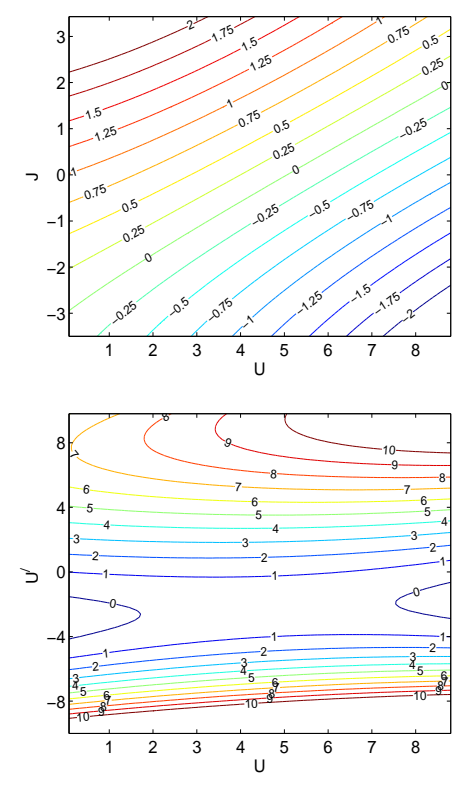

FIG. 4: The determinants of magnetic instabilities for $J$ vs. $U$ and $U^{\prime}$ vs. $U$. The contour line $-0-0-$ gives the parameter groups of instability.

exists between different bands a charge-orbital instability may occur. Apart from this region the charge-orbital susceptibility also drops rapidly. It is worthy to notice that the charge-orbital susceptibility depends weakly on the value of $U$, the intra-band repulsion, except for the case of very strong inter-band repulsion.

The SDW phase has striking temperature dependence. It is found that at higher temperatures a new resonance appears at the $\Gamma$ point, which starts to increase rapidly at a temperature $k T \sim 0.08$, as shown in Fig.5. This resonance corresponds to the formation of a paramagnetic phase in the material. The SDW peak at the M point drops to a nearly stable value at this temperature. This result is qualitatively in agreement with the experimental observation for the stripe-like AFM phase in $\mathrm{LaOFeAs}$, which forms under a temperature 134K[16]. At $k T_{c}=0.12$ the intensity of paramagnetic phase starts to surpass that of the striped AFM phase. It is found that temperature changes hardly the bare spin susceptibility but smooths its distribution in the BZ. Reducing the intra-band coupling parameter $U$, the relative intensity between the paramagnetic phase and the SDW phase reduces significantly. Hence it is confirmed that this new resonance comes from the intra-band coupling. Therefore, this temperature dependence of the spin susceptibility provides detailed information for the band structure of the oxypnictide material. More experimental evidences for this temperature dependence are expected. At very low temperatures the spin susceptibility showed more abundant structures but requires further stringent calculations.

We tried to solve the superconducting gap equation by including a pairing coupling term into the interac- 

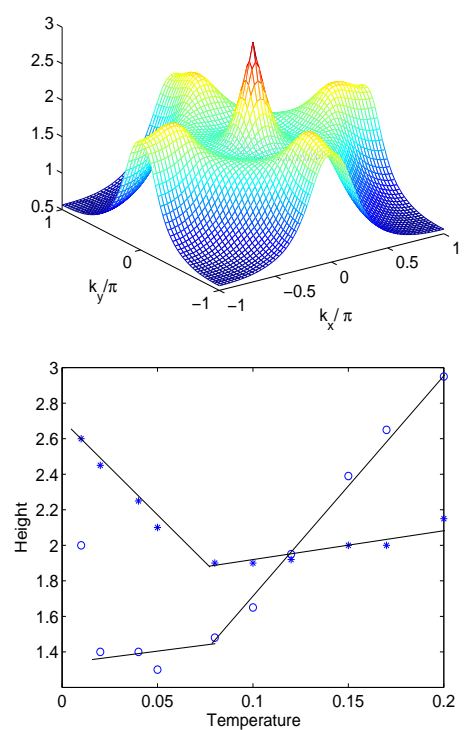

FIG. 5: Spin susceptibility with temperature $T=0.2$ (upper) and temperature dependence of the intensities of spin susceptibility at the $\Gamma$ point(circles) and $M$ point(stars) in the $\mathrm{BZ}$ (lower). Both results take $U=3.0, J=0, U^{\prime}=1.0$. Lines are guides for the eye.

tion Hamiltonian but failed to find a stable gap function with some symmetries, such as $s, s^{-}, d_{x^{2}-y^{2}}, d_{x y}$, etc. This failure also exists in some literatures, e.g., Yao et al found only eigenvalues $0.1-0.4$ with the $d_{x y}$-wave symmetry [17]. But Yanagi et al claimed that some groups of interaction parameters give eigenvalue unity for this gap symmetry in the same material 18]. Therefore, the gap symmetry remains controversy and requires further studies.

In conclusion, a three-band model is set up to reproduce the band structure near the fermi surfaces of oxypnictide. It shows a hole pocket around the $\Gamma$ point and a electron pocket around $\mathrm{M}$ point in the extended BZ. The spin susceptibility with various interactions are calculated in the random phase approximation. It is found that the spin susceptibility peaks around the $\mathrm{M}$ point, showing a SDW with momentum $(0, \pi)$ and a clear stripelike spin arrangement. The intra-band coupling enhances remarkably the SDW but the Hund's coupling weakens it. The interaction parameters are determined for the magnetic instability in oxypnictide. Finally the temperature dependence of the spin susceptibility is studied. It is found that a new resonance appears at higher temperatures at the $\Gamma$ point indicating the formation of a paramagnetic phase. There is a transition between the SDW phase and the paramagnetic phase.

This work was supported by the National Natural Science Foundation of China (Grant No. 10874049), the State Key Program for Basic Research of China (No. 2007CB925204) and the Natural Science Foundation of Guangdong province ( No. 07005834 ).
[1] Y. Kamihara, T. Watanabe, M. Hirano, and H. Hosonno, J. Am. Chem. Soc. 130, 3296 (2008).

[2] X. H. Chen, T. Wu, G. Wu, R. H. Liu, H. Chen, and D. F. Fang, Nature 453, 761 (2008).

[3] J. dong, H. J. Zhang, G. Xu, eta al, Europhys. Lett. 83, 27006 (2008).

[4] I. I. Mazin, D. J. Singh, M. D. Johannes, and M. H. Du, Phys. Rev. Lett. 101, 057003 (2008).

[5] C. de la Cruz, Q. Huang, J. W. Lynn, eta al, Nature 453 , 899 (2008).

[6] G. F. Chen, Z. Li, D. Wu, eta al, Phys. Rev. Lett. 100, 247002 (2008).

[7] M. A. McGuire, A. D. Christianson, A. S. Sefat, eta al, arXiv:0804.0796 [cond-mat.supr-con].

[8] D. Singh and M. H. Du, arXiv:0803.0429 [cond-mat.suprcon].

[9] L. Boeri, O. V. Dolgov, and A. A. Golubov, Phys. Rev. Lett. 101, 026404 (2008).
[10] K. Haule, J. H. Shim, and G. Kotliar , Phys. Rev. Lett. 100, 226402 (2008).

[11] Patrick. A. Lee, and X. G. Wen, Phys. Rev. B 78, 144517 (2008).

[12] S. L. Yu, J. Kang, and J. X. Li, Phys. Rev. B 79, 064517 (2009).

[13] T. Yildirim, arXiv:0804.2252 [cond-mat.supr-con].

[14] T. Takimoto, T. Hotta, and K. Ueda, Phys. Rev. B 69, 104504 (2004).

[15] S. Raghu, X. L. Qi, C. X. Liu, D. J. Scalapino, and S. C. Zhang, Phys. Rev. B 77, 220503(R) (2008).

[16] Clarina de la Cruz 1,2, Q. Huang3, J. W. Lynn3, arXiv:0804.0795 cond-mat.supr-con].

[17] Z. J. Yao, J. X. Li, and Z. D. Wang, arXiv:0804.4166 [cond-mat.supr-con].

[18] Yuki Yanagi, Youichi Yamakawa, and Yoshiaki ONO, J. of the Phys. Soc. of Japan, 77, 123701 (2008). 